\title{
Efficient Asymmetric Synthesis of Prostaglandin $\mathbf{E}_{1}$
}

\author{
Jae-Chul Jung ${ }^{\mathrm{a}}$ and Oee-Sook Park ${ }^{\mathrm{b}}$ \\ a Department of Medicinal Chemistry, School of Pharmacy, University of Mississippi, P. O. Box \\ 1848, University, Mississippi 38677-1848, USA \\ ${ }^{b}$ Department of Chemistry, College of Natural Sciences, Chungbuk National University, \\ Cheongju 361-763, Chungbuk, South Korea \\ Reprint requests to Dr. Oee-Sook Park. Fax: +82-43-267-2279. E-mail: ospark@cbnu.ac.kr
}

Z. Naturforsch. 2007, 62b, 556-560; received July 31, 2006

\begin{abstract}
A simple synthesis of prostaglandin $\mathrm{E}_{1}\left(\mathrm{PGE}_{1}\right)$ is described. The key steps are an asymmetric Michael addition to establish the desired $(R)$-configurations at $\mathrm{C} 8$ and $\mathrm{C} 12$ of the 2(trimethylsilyl)ethoxymethyl- (SEM) protected $\mathrm{PGE}_{1}$ and its one-pot deprotection with magnesium bromide in high yield. This method is potentially useful for the preparation of other modified prostaglandins.
\end{abstract}

Key words: Prostaglandin, Asymmetric Michael Addition, Cuprates, Deprotection

\section{Introduction}

Prostaglandin and its analogues have important functions in the animal body and a variety of biological effects [1]. Many naturally occurring prostaglandins as well as many artificial forms have been synthesized by several groups [2]. The major approaches to the synthesis of prostaglandins fall into three categories (Fig. 1). Key steps in the original approach included a Wittig reaction of aldehyde 2 and triphenylphosphane (3), followed by a Wittig-Horner reaction to furnish the C13-C14 E-olefin partial structure of prostaglandins [3]. Another strategy employed Michael addition reactions of ester $\mathbf{5}$ and allylic alcohol 6 [4]. In an alternative approach, the Noyori reaction of cyclopentenone $\mathbf{7}$ with allylic alcohol $\mathbf{6}$ and $Z$-allylic iodide 8 readily generated prostaglandins, which is the most elegant synthetic method in the area of three component coupling [5].

Since the first isolation of prostaglandins, numerous synthetic methods have been developed [6]. Most of these routes are based on two or three component coupling reactions. Recently, Feringa reported an enantioselective synthesis using a tandem 1,4-addition aldol reaction to a cyclopentene3,5-dione monoacetal [7]. Florent generated a chiral amino-cyclopentenone moiety via [3.3] sigmatropic rearrangement and palladium-catalyzed crosscoupling followed by a ring closing metathesis (RCM) reaction [8].
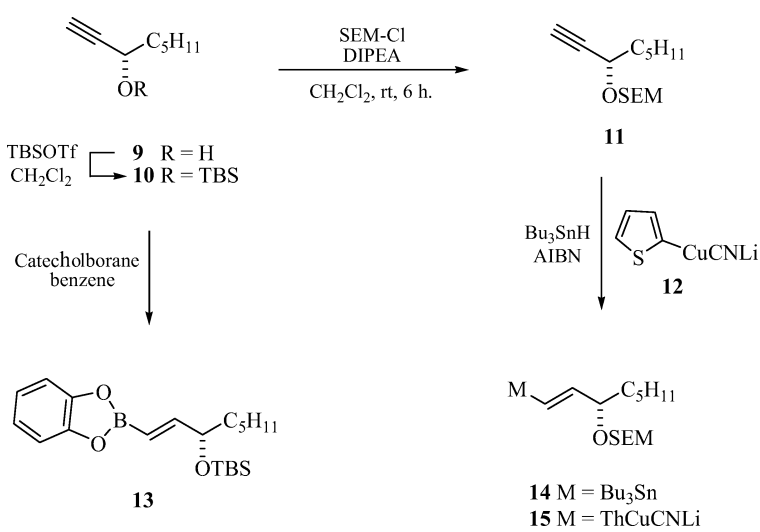

Scheme 1. Synthesis of key fragments $\mathbf{1 3}$ and $\mathbf{1 5 .}$

During our studies of asymmetric Michael addition reactions of cyclopentenones, we realized that a more efficient method of preparing prostaglandin $E_{1}$ $\left(\mathrm{PGE}_{1}, \mathbf{1}\right)$ and its analogues was needed. Herein, we describe an efficient synthesis of prostaglandin $E_{1}$ (1) via asymmetric Michael addition employing 2-(trimethylsilyl)ethoxymethyl (SEM) protecting groups.

\section{Results and Discussion}

Synthesis of fragments $\mathbf{1 3}$ and 14 required the development of a method for preparing tertbutyldimethylsilyl (TBS) or SEM-protected ethers 10 and 11 in excellent yields (Scheme 1). These were ac- 
Suzuki coupling Wittig reaction

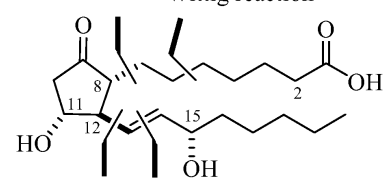

Michael addition Wittig-Horner

olefination

$1 \mathrm{PGE}_{1}$<smiles>O=C(O)CCCCCCC1=CCCC1=O</smiles>

$\mathrm{HO}$

16

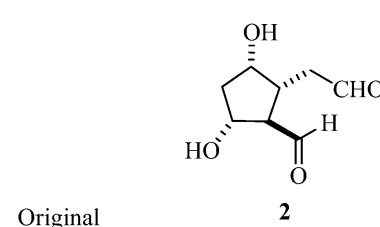

synthesis

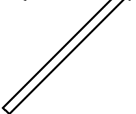
coupling $\stackrel{\longrightarrow}{\longrightarrow}$

Three component

coupling
$\mathrm{Ph}_{3} \mathrm{P} \sim \mathrm{CO}_{2}^{-}$

3

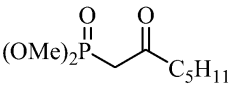

4<smiles>COC(=O)CCCCCCC1=C[C@@H](C)CC1=O</smiles><smiles>[X]C=C[C@@H](O)CCCCC</smiles>

$6 \mathrm{X}=\mathrm{I}$ or $\mathrm{ThCuCNLi}$
Fig. 1. General synthetic approaches to prostaglandins.<smiles>CCCCC[C@H](O)/C=C/C=C/CCCC(C)=O</smiles>

$\underset{\mathrm{ClCH}_{2} \mathrm{OCH}_{2} \mathrm{CH}_{2} \mathrm{SiMe}_{3}}{\stackrel{\mathrm{Li}_{2} \mathrm{CO}_{3}, \text { DMAP }}{\text { DMF, rt, } 20 \mathrm{~h} .}}$
. SEMO<smiles>[M]OC(=O)CCCCCCC1=C[C@@H](I)CC1=O</smiles>

17<smiles>CCCCCCCCCC1C(=O)C[C@H](O)C1/C=C\[C@H](O)CCCCCC(=O)O</smiles>

$1 \mathrm{PGE}_{1}$

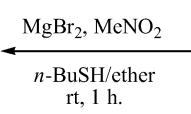

$\mathrm{rt}, 1 \mathrm{~h}$.

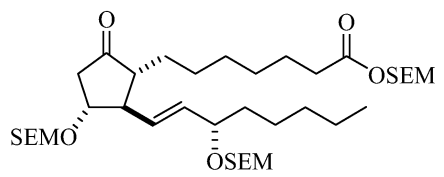

18
Scheme 2. Synthesis of prostaglandin $\mathrm{E}_{1}(\mathbf{1})$. complished by subjecting commercially available $(S)$ (-)-1-octyn-3-ol (9) to TBS or SEM protection. Fragment 13 was smoothly prepared from TBS-ether 10 which underwent hydroboration with catecholborane in benzene in $80 \%$ yield [9]. E-Vinylstannane $\mathbf{1 4}$ was obtained from SEM-ether 11 through hydrostan- nation with tributyltin hydride $\left(\mathrm{Bu}_{3} \mathrm{SnH}\right)$ in the presence of $2,2^{\prime}$-azo-bis-isobutyronitrile (AIBN) as an initiator in $70 \%$ yield [10]. Bis-protection of the commercially available chiral hydroxyl acid 16 [11] with 2 equiv. of SEM-Cl in the presence of lithium carbonate $\left(\mathrm{Li}_{2} \mathrm{CO}_{3}\right)$ and 4-(dimethylamino)pyridine (DMAP) 
gave SEM-cyclopentenone 17. Asymmetric Michael addition of 17 with $E$-vinylstannane 14 [12] in the presence of $n$-BuLi at $-78{ }^{\circ} \mathrm{C}$ in THF gave fully protected SEM-ester $\mathbf{1 8}$ in $67 \%$ yield. However, treatment of 17 with vinylborane $\mathbf{1 3}$ was unsatisfactory, and for the most part, the starting material was recovered. Finally, treatment of fully protected SEMester 18 with magnesium bromide and nitromethane in the presence of 1-butanethiol [13] led to prostaglandin $E_{1}$ (1) (Scheme 2), whose spectral and physical data were identical to those reported [14].

In conclusion, the asymmetric synthesis of prostaglandin $\mathrm{E}_{1}(\mathbf{1})$ was accomplished in a three-step process. The use of asymmetric conjugated addition to construct $(R)$-configurations at $\mathrm{C} 8, \mathrm{C} 12$ and an efficient deprotection of fully protected SEM-ester $\mathbf{1 8}$ under mild reaction conditions provided an economical and practical method for the preparation of prostaglandins.

\section{Experimental Section}

Reactions requiring anhydrous conditions were performed with the usual precautions for rigorous exclusion of air and moisture. Tetrahydrofuran was distilled from sodium benzophenone ketyl prior to use. Thin layer chromatography (TLC) was performed on precoated silica gel G and GP uniplates from Analtech and visualized with $254 \mathrm{~nm}$ UV light. Flash chromatography was carried out on silica gel 60 [Scientific Adsorbents Incorporated (SAI), particle size 32 $63 \mu \mathrm{m}$, pore size $60 \AA$ ]. ${ }^{1} \mathrm{H} \mathrm{NMR},{ }^{13} \mathrm{C} \mathrm{NMR}$, and 2D NMR spectra were recorded on Bruker DPX 400, 500 instruments at $400 \mathrm{MHz}, 500 \mathrm{MHz}$ and $100 \mathrm{MHz}, 125 \mathrm{MHz}$, respectively. The chemical shifts are reported in parts per million (ppm) downfield from tetramethylsilane, and $J$ values are in Hz. Infrared (IR) spectra were obtained on an ATI Mattson FT/IR spectrometer. Mass spectra were recorded with a Waters Micromass ZQ LC-Mass system and high-resolution mass spectra (HRMS) were measured with a Bruker BioApex FTMS system by direct injection using an electrospray interface (ESI). When necessary, chemicals were purified according to the reported procedures [15].

\section{(S)-3-(2-Trimethylsilylethoxymethoxy)oct-1-yne (11)}

To a stirred solution of (S)-(-)-1-octyn-3-ol (9, $0.26 \mathrm{~g}$, $2.0 \mathrm{mmol})$ in dry $\mathrm{CH}_{2} \mathrm{Cl}_{2}(5 \mathrm{~mL})$ was added DIPEA $(0.39 \mathrm{~g}$, $3.0 \mathrm{mmol}$ ) under argon atmosphere at $0{ }^{\circ} \mathrm{C}$, followed by addition of SEM-Cl $(0.4 \mathrm{~g}, 2.4 \mathrm{mmol})$. The reaction mixture was stirred at r.t. for $6 \mathrm{~h}$, and quenched with saturated aqueous $\mathrm{NH}_{4} \mathrm{Cl}$ solution $(2 \mathrm{~mL})$. The organic phase was separated and washed with water $(2 \mathrm{~mL})$ and brine $(2 \mathrm{~mL})$. The organic layer was separated, dried over anhydrous $\mathrm{MgSO}_{4}$, filtered and concentrated under reduced pressure. The crude product was purified by flash column chromatography (silica gel, $3 \%$ EtOAc/hexanes) to afford SEM-ether 11 (0.5 g, $98 \%)$ as a colorless oil. $R_{f}=0.5$ (3\% ethyl acetate in hexanes). $-[\alpha]_{\mathrm{D}}^{24}=-128.3\left(c=1.1, \mathrm{CHCl}_{3}\right)$. - IR (neat, $\left.\mathrm{NaCl}\right): v=$ 3312, 2954, 2894, 1647, 1249, 1024, $836 \mathrm{~cm}^{-1} .-{ }^{1} \mathrm{H}$ NMR $\left(\mathrm{CDCl}_{3}, 500.14 \mathrm{MHz}\right): \delta=4.93\left(\mathrm{~d}, J=6.8 \mathrm{~Hz}, 1 \mathrm{H}, \mathrm{OCH}_{2} \mathrm{O}\right)$, $4.67\left(\mathrm{~d}, J=6.8 \mathrm{~Hz}, 1 \mathrm{H}, \mathrm{OCH}_{2} \mathrm{O}\right), 4.33(\mathrm{t}, J=6.4 \mathrm{~Hz}, 1 \mathrm{H}$, $\mathrm{CH}), 3.70\left(\mathrm{q}, J=10.4 \mathrm{~Hz}, 1 \mathrm{H}, \mathrm{CH}_{2}\right), 3.53(\mathrm{q}, J=6.4 \mathrm{~Hz}, 1 \mathrm{H}$, $\left.\mathrm{CH}_{2}\right), 2.44$ (brs, $\left.1 \mathrm{H}, \mathrm{CH}\right), 1.75-1.69\left(\mathrm{~m}, 2 \mathrm{H}, \mathrm{CH}_{2}\right), 1.50-$ $1.41\left(\mathrm{~m}, 2 \mathrm{H}, \mathrm{CH}_{2}\right), 1.34-1.26\left(\mathrm{~m}, 4 \mathrm{H}, 2 \times \mathrm{CH}_{2}\right), 1.00-$ $0.92\left(\mathrm{~m}, 2 \mathrm{H}, \mathrm{CH}_{2}\right), 0.89$ (t, $\left.J=6.8 \mathrm{~Hz}, 3 \mathrm{H}, \mathrm{CH}_{3}\right), 0.01$ (s, $\left.9 \mathrm{H}, \mathrm{SiMe}_{3}\right) .-{ }^{13} \mathrm{C} \mathrm{NMR}\left(\mathrm{CDCl}_{3}, 125.67 \mathrm{MHz}\right): \delta=92.4$ $\left(\mathrm{OCH}_{2} \mathrm{O}\right), 82.8(\mathrm{C}), 73.2(\mathrm{CH}), 65.4(\mathrm{CH}), 65.3\left(\mathrm{CH}_{2}\right), 36.0$ $\left(\mathrm{CH}_{2}\right), 31.5\left(\mathrm{CH}_{2}\right), 24.9\left(\mathrm{CH}_{2}\right), 22.6\left(\mathrm{CH}_{2}\right), 18.1\left(\mathrm{CH}_{2}\right)$, $14.0\left(\mathrm{CH}_{3}\right),-1.4\left(\mathrm{SiMe}_{3}, 3 \mathrm{C}\right)$. - HRMS: $\mathrm{m} / z=257.1944$ (calcd. 257.1937 for $\mathrm{C}_{14} \mathrm{H}_{29} \mathrm{O}_{2} \mathrm{Si},[\mathrm{M}+\mathrm{H}]^{+}$).

\section{(S)-3-(2-Trimethylsilylethoxymethoxy)-1-tributylstannyl-1- octene (14)}

To a stirred solution of SEM-ether $11(0.51 \mathrm{~g}, 2.0 \mathrm{mmol})$, tributyltin hydride $(0.88 \mathrm{~g}, 3.0 \mathrm{mmol})$ and 2,2'-azo-bisisobutyronitrile (4 mg, $0.024 \mathrm{mmol})$ were added under argon atmosphere and kept for $1 \mathrm{~h}$ at $120{ }^{\circ} \mathrm{C}$. The reaction mixture was allowed to cool to r.t. The crude product was purified by flash column chromatography (silica gel, $1 \%$ EtOAc/hexanes) to afford $E$-vinylstannane $14(0.77 \mathrm{~g}$, $70 \%)$ as a pale yellow oil. $\mathrm{R}_{f}=0.4$ (1\% ethyl acetate in hexanes): $-[\alpha]_{\mathrm{D}}^{25}=-83.0\left(c=0.6, \mathrm{CHCl}_{3}\right)$. - IR (neat, $\mathrm{NaCl}): v=2956,2873,1464,1377,1249,1054,836 \mathrm{~cm}^{-1}$. $-{ }^{1} \mathrm{H} \mathrm{NMR}\left(\mathrm{CDCl}_{3}, 500.14 \mathrm{MHz}\right) \delta=6.11(\mathrm{~d}, J=19.2 \mathrm{~Hz}$, $1 \mathrm{H}, \mathrm{CH}=\mathrm{CH}), 5.78(\mathrm{dd}, J=7.2,18.8 \mathrm{~Hz}, 1 \mathrm{H}, \mathrm{CH}=\mathrm{CH})$, $4.68\left(\mathrm{dd}, J=6.8,6.8 \mathrm{~Hz}, 2 \mathrm{H}, \mathrm{OCH}_{2} \mathrm{O}\right), 4.00-3.95(\mathrm{~m}, 1 \mathrm{H}$, $\mathrm{CH}), 3.80-3.73\left(\mathrm{~m}, 1 \mathrm{H}, \mathrm{CH}_{2}\right), 3.56-3.49\left(\mathrm{~m}, 1 \mathrm{H}, \mathrm{CH}_{2}\right)$, $1.66-1.55\left(\mathrm{~m}, 2 \mathrm{H}, \mathrm{CH}_{2}\right), 1.52-1.43\left(\mathrm{~m}, 6 \mathrm{H}, 3 \times \mathrm{CH}_{2}\right)$, $1.38-1.25\left(\mathrm{~m}, 12 \mathrm{H}, 6 \times \mathrm{CH}_{2}\right), 1.00-0.81(\mathrm{~m}, 20 \mathrm{H}, 4 \times$ $\left.\mathrm{CH}_{3}, 4 \times \mathrm{CH}_{2}\right), 0.04$ (s, 9H, $\left.\mathrm{SiMe}_{3}\right),-{ }^{13} \mathrm{C} \mathrm{NMR}\left(\mathrm{CDCl}_{3}\right.$, 125.67 MHz): $\delta=148.5(\mathrm{CH}=\mathrm{CH}), 131.1(\mathrm{CH}=\mathrm{CH}), 91.8$ $\left(\mathrm{OCH}_{2} \mathrm{O}\right), 79.9(\mathrm{CH}), 65.0\left(\mathrm{CH}_{2}\right), 35.3\left(\mathrm{CH}_{2}\right), 31.8\left(\mathrm{CH}_{2}\right)$, $29.1\left(3 \times \mathrm{CH}_{2}, 3 \mathrm{C}\right), 27.4\left(3 \times \mathrm{CH}_{2}, 3 \mathrm{C}\right), 25.2\left(\mathrm{CH}_{2}\right), 22.7$ $\left(\mathrm{CH}_{2}\right), 18.1\left(\mathrm{CH}_{2}\right), 14.1\left(\mathrm{CH}_{3}\right), 13.7\left(3 \times \mathrm{CH}_{3}, 3 \mathrm{C}\right), 9.5$ $\left(3 \times \mathrm{CH}_{2}, 3 \mathrm{C}\right),-1.4\left(\mathrm{SiMe}_{3}, 3 \mathrm{C}\right) .-\mathrm{HRMS}: \mathrm{m} / \mathrm{z}=549.3158$ (calcd. 549.3150 for $\mathrm{C}_{26} \mathrm{H}_{57} \mathrm{O}_{2} \mathrm{SiSn},[\mathrm{M}+\mathrm{H}]^{+}$).

\section{2-Trimethylsilylethoxymethyl-(R)-3-(2-trimethylsilylethoxy- methoxy)-5-oxo-1-cyclopentene-1-heptanoate (17)}

To a stirred solution of acid $16(0.23 \mathrm{~g}, 1.0 \mathrm{mmol})$ in dry DMF $(3 \mathrm{~mL})$ were added DMAP $(3 \mathrm{mg}, 0.02 \mathrm{mmol})$ and lithium carbonate $(0.16 \mathrm{~g}, 2.2 \mathrm{mmol})$ under argon atmosphere at $10{ }^{\circ} \mathrm{C}$, followed by addition of SEM-Cl $(0.37 \mathrm{~g}$, $2.2 \mathrm{mmol}$ ) at that temperature. The reaction mixture was stirred at r.t. for $20 \mathrm{~h}$. The reaction mixture was diluted with ether $(10 \mathrm{~mL})$ and washed with water $(5 \mathrm{~mL})$ and 
brine $(5 \mathrm{~mL})$. The organic layer was separated, dried over anhydrous $\mathrm{MgSO}_{4}$, filtered and concentrated under reduced pressure. The crude product was purified by flash column chromatography (silica gel, $15 \%$ EtOAc/hexanes) to afford SEM-cyclopentenone $\mathbf{1 7}(0.15 \mathrm{~g}, 31 \%)$ as a pale yellow oil. $\mathrm{R}_{f}=0.4$ (15\% ethyl acetate in hexanes). $-[\alpha]_{\mathrm{D}}^{25}=-4.0$ $\left(c=0.2, \mathrm{CHCl}_{3}\right)$. - IR (neat, $\left.\mathrm{NaCl}\right): v=2951,2898,1740$ (CO), 1715 (COO), 1408, 1249, 1032, $837 \mathrm{~cm}^{-1} .-{ }^{1} \mathrm{H}$ NMR $\left(\mathrm{CDCl}_{3}, 500.14 \mathrm{MHz}\right): \delta=7.18(\mathrm{~s}, 1 \mathrm{H}, \mathrm{CH}=\mathrm{CH}), 5.27$, (s, $\left.2 \mathrm{H}, \mathrm{OCH}_{2} \mathrm{O}\right), 4.78(\mathrm{~d}, J=3.2 \mathrm{~Hz}, 1 \mathrm{H}, \mathrm{CH}), 4.76(\mathrm{~s}, 2 \mathrm{H}$, $\left.\mathrm{OCH}_{2} \mathrm{O}\right), 3.71-3.61\left(\mathrm{~m}, 4 \mathrm{H}, 4 \times \mathrm{OCH}_{2}\right), 2.76(\mathrm{dd}, J=6.0$, $\left.18.8 \mathrm{~Hz}, 1 \mathrm{H}, \mathrm{CH}_{2}\right), 2.35\left(\mathrm{dd}, J=1.6,17.6 \mathrm{~Hz}, 1 \mathrm{H}, \mathrm{CH}_{2}\right)$, $3.23\left(\mathrm{t}, J=7.6 \mathrm{~Hz}, 2 \mathrm{H}, \mathrm{CH}_{2}\right), 2.18\left(\mathrm{t}, J=7.6 \mathrm{~Hz}, 2 \mathrm{H}, \mathrm{CH}_{2}\right)$, $1.67-1.59\left(\mathrm{~m}, 2 \mathrm{H} \mathrm{CH}_{2}\right), 1.53-1.46\left(\mathrm{~m}, 2 \mathrm{H}, \mathrm{CH}_{2}\right), 1.37-$ $1.31\left(\mathrm{~m}, 4 \mathrm{H}, 4 \times \mathrm{CH}_{2}\right), 0.96\left(\mathrm{t}, J=8.4 \mathrm{~Hz}, 4 \mathrm{H}, 4 \times \mathrm{SiCH}_{2}\right)$, 0.03 (s, 9H, $\mathrm{SiMe}_{3}$ ), 0.02 (s, 9H, $\left.\mathrm{SiMe}_{3}\right) .-{ }^{13} \mathrm{C} \mathrm{NMR}$ $\left(\mathrm{CDCl}_{3}, 125.67 \mathrm{MHz}\right): \delta=205.9(\mathrm{CO}), 173.3(\mathrm{COO}), 154.4$ $(\mathrm{CH}), 148.3(\mathrm{C}), 94.5\left(\mathrm{CH}_{2}\right), 88.7\left(\mathrm{CH}_{2}\right), 73.6(\mathrm{CH}), 67.8$ $\left(\mathrm{CH}_{2}\right), 65.5\left(\mathrm{CH}_{2}\right), 42.7\left(\mathrm{CH}_{2}\right), 34.3\left(\mathrm{CH}_{2}\right), 29.0\left(\mathrm{CH}_{2}\right)$, $28.8\left(\mathrm{CH}_{2}\right), 27.2\left(\mathrm{CH}_{2}\right), 24.7\left(\mathrm{CH}_{2}\right), 24.5\left(\mathrm{CH}_{2}\right), 18.1$ $\left(\mathrm{CH}_{2}\right), 18.0\left(\mathrm{CH}_{2}\right),-1.2\left(\mathrm{SiMe}_{3}, 3 \mathrm{C}\right),-1.4\left(\mathrm{SiMe}_{3}, 3 \mathrm{C}\right)$. HRMS: $m / z=487.2903$ (calcd. 487.2911 for $\mathrm{C}_{24} \mathrm{H}_{47} \mathrm{O}_{6} \mathrm{Si}_{2}$, $\left.[\mathrm{M}+\mathrm{H}]^{+}\right)$.

\section{Preparation of 2-thienyl(cyano)copper lithium (12, 2-ThCuCNLi)}

To a stirred solution of thiophene $(1.56 \mathrm{~g}, 20.4 \mathrm{mmol})$ in THF (10 mL) $n$-BuLi (12.5 mL, $20.0 \mathrm{mmol}$; $1.6 \mathrm{M}$ solution in hexane) was added at $-78{ }^{\circ} \mathrm{C}$ under argon atmosphere, and stirring was continued at $-78^{\circ} \mathrm{C}$ for $30 \mathrm{~min}$. The mixture was transferred to a slurry of copper cyanide $(1.8 \mathrm{~g}, 20.0 \mathrm{mmol})$ in THF $(10 \mathrm{~mL})$. The resulting reaction mixture was warmed to $-40{ }^{\circ} \mathrm{C}$ and diluted with THF $(66 \mathrm{~mL})$ to give a clear solution $(0.2 \mathrm{M}$ solution in THF), which was stored in a freezer at $-22{ }^{\circ} \mathrm{C}$.

(8R,11R,12R,15S)-11,15-Bis-(2-trimethylsilylethoxymethoxy)-9-oxo-prost-13-en-1-oic acid 2-trimethylsilylethoxymethyl ester (18)

A solution of vinylstannane $\mathbf{1 4}(0.12 \mathrm{~g}, 0.22 \mathrm{mmol})$ in dry THF $(0.5 \mathrm{~mL})$ was treated with $n$ - $\operatorname{BuLi}(0.15 \mathrm{~mL}, 0.22 \mathrm{mmol}$;
$1.6 \mathrm{M}$ solution in hexane) at $-78{ }^{\circ} \mathrm{C}$ under argon atmosphere. The mixture was stirred at $-78{ }^{\circ} \mathrm{C}$ for $30 \mathrm{~min}$, and a solution of ThCuCNLi $(1.4 \mathrm{~mL}, 0.28 \mathrm{mmol} ; 0.2 \mathrm{M}$ solution in THF) in THF $(0.5 \mathrm{~mL})$ was added. The reaction mixture was stirred at that temperature for $30 \mathrm{~min}$, and a solution of SEM-cyclopentenone $17(0.1 \mathrm{~g}, 0.2 \mathrm{mmol})$ in THF $(1 \mathrm{~mL})$ was added. The resulting mixture was stirred at $-78{ }^{\circ} \mathrm{C}$ for $30 \mathrm{~min}$, and quenched with $10 \%$ aqueous $\mathrm{NH}_{4} \mathrm{OH}$ solution $(2 \mathrm{~mL})$ and saturated aqueous $\mathrm{NH}_{4} \mathrm{Cl}$ solution $(3 \mathrm{~mL})$. The mixture was warmed to r.t., diluted with ether $(10 \mathrm{~mL})$ and washed with brine $(5 \mathrm{~mL})$. The organic layer was separated, dried over anhydrous $\mathrm{MgSO}_{4}$, filtered and concentrated under reduced pressure. The crude product was purified by flash column chromatography (silica gel, $20 \%$ ether/hexanes) to afford fully protected SEM ester $\mathbf{1 8}$ $(0.1 \mathrm{~g}, 67 \%)$ as a pale yellow oil. $R_{f}=0.3(20 \%$ ether in hexanes). $-[\alpha]_{\mathrm{D}}^{25}=+30.7\left(c=1.2, \mathrm{CHCl}_{3}\right)$. - IR (neat, $\mathrm{NaCl}): v=2951,1740(\mathrm{CO}), 1716(\mathrm{COO}), 1463,1249,1105$, 1031, $836 \mathrm{~cm}^{-1} .{ }^{1} \mathrm{H}$ NMR $\left(\mathrm{CDCl}_{3}, 500.14 \mathrm{MHz}\right): \delta=$ $6.96(\mathrm{dd}, J=5.0,3.8 \mathrm{~Hz}, 1 \mathrm{H}, \mathrm{CH}=\mathrm{CH}), 6.80(\mathrm{dd}, J=0.8$, $3.4 \mathrm{~Hz}, 1 \mathrm{H}, \mathrm{CH}=\mathrm{CH}), 5.29\left(\mathrm{~s}, 2 \mathrm{H}, \mathrm{OCH}_{2} \mathrm{O}\right), 5.28(\mathrm{~s}, 2 \mathrm{H}$, $\left.\mathrm{OCH}_{2} \mathrm{O}\right), 4.80\left(\mathrm{~d}, J=2.4 \mathrm{~Hz}, 2 \mathrm{H}, \mathrm{OCH}_{2} \mathrm{O}\right), 4.74(\mathrm{~d}, J=$ $7.2 \mathrm{~Hz}, 1 \mathrm{H}, \mathrm{CH}), 3.74-3.67\left(\mathrm{~m}, 6 \mathrm{H}, 3 \times \mathrm{CH}_{2}\right), 3.66-3.60$ $(\mathrm{m}, 1 \mathrm{H}, \mathrm{CH}), 2.81$ (ddd, $J=6.8,6.4,6.0 \mathrm{~Hz}, 2 \mathrm{H}, \mathrm{CH}_{2}$, $\mathrm{CH}), 2.40-2.28\left(\mathrm{~m}, 4 \mathrm{H}, \mathrm{CH}_{2}, \mathrm{CH}\right), 2.03(\mathrm{t}, J=11.5 \mathrm{~Hz}$, $\left.2 \mathrm{H}, \mathrm{CH}_{2}\right), 1.67-1.59\left(\mathrm{~m}, 4 \mathrm{H}, 2 \times \mathrm{CH}_{2}\right), 1.56-1.47(\mathrm{~m}, 4 \mathrm{H}$, $\left.2 \times \mathrm{CH}_{2}\right), 1.40-1.28\left(\mathrm{~m}, 8 \mathrm{H}, 4 \times \mathrm{CH}_{2}\right), 1.01-0.93(\mathrm{~m}, 9 \mathrm{H}$, $\left.3 \times \mathrm{CH}_{2}, \mathrm{CH}_{3}\right), 0.05$ (s, 3H, SiMe 3 ), 0.04 (s, 6H, SiMe $)$, 0.04 (s, 9H, $\left.\mathrm{SiMe}_{3}\right), 0.03$ (s, 9H, $\left.\mathrm{SiMe}_{3}\right) .-{ }^{13} \mathrm{C} \mathrm{NMR}$ $\left(\mathrm{CDCl}_{3}, 125.67 \mathrm{MHz}\right): \delta=206.0(\mathrm{CO}), 173.5(\mathrm{COO}), 154.5$ $(\mathrm{CH}=\mathrm{CH}), 148.6(\mathrm{CH}=\mathrm{CH}), 94.7\left(\mathrm{CH}_{2}\right), 93.7\left(\mathrm{CH}_{2}\right), 89.0$ $\left(\mathrm{CH}_{2}\right), 84.9(\mathrm{CH}), 78.7(\mathrm{CH}), 73.7(\mathrm{CH}), 68.0\left(\mathrm{CH}_{2}\right), 65.8$ $\left(\mathrm{CH}_{2}\right), 65.4\left(\mathrm{CH}_{2}\right), 51.6(\mathrm{CH}), 42.9\left(\mathrm{CH}_{2}\right), 34.5\left(2 \times \mathrm{CH}_{2}\right.$, 2C), $29.3\left(\mathrm{CH}_{2}\right), 29.2\left(\mathrm{CH}_{2}\right), 29.1\left(\mathrm{CH}_{2}\right), 29.0\left(\mathrm{CH}_{2}\right), 27.7$ $\left(\mathrm{CH}_{2}\right), 27.5\left(\mathrm{CH}_{2}\right), 26.2\left(\mathrm{CH}_{2}\right), 24.9\left(\mathrm{CH}_{2}\right), 24.7\left(\mathrm{CH}_{2}\right)$, $18.3\left(\mathrm{CH}_{2}\right), 18.2\left(2 \times \mathrm{CH}_{2}, 2 \mathrm{C}\right),-1.2\left(3 \times \mathrm{SiMe}_{3}, 9 \mathrm{C}\right)$. HRMS: $m / z=745.4932$ (calcd. 745.4926 for $\mathrm{C}_{38} \mathrm{H}_{76} \mathrm{O}_{8} \mathrm{Si}_{3}$, $\left.[\mathrm{M}+\mathrm{H}]^{+}\right)$.

\section{Acknowledgement}

This work was supported by a Chungbuk National University Grant in 2005.
[1] P.W. Collins, S.W. Djuric, Chem. Rev. 1993, 93, $1533-1564$.

[2] C. J. Sih, P. Price, R. Sood, R. G. Salomon, G. Peruzzotti, M. Casey, J. Am. Chem. Soc. 1972, 94, $3642-$ 3644; F. Sato, H. Tsujiyama, N. Ono, T. Yoshino, S. Okamuko, Tetrahedron Lett. 1990, 31, $4481-4484$; O. W. Gooding, C. C. Beard, G. F. Cooper, D. Y. Jackson, J. Org. Chem. 1993, 58, 3681-3686.

[3] E. J. Corey, N. M. Weinshenker, T. K. Schaaf, W. Huber, J. Am. Chem. Soc. 1969, 91, 5675-5677.
[4] F. S. Alvarez, D. Wren, A. Prince, J. Am. Chem. Soc. 1972, 94, 7823-7827.

[5] R. Noyori, M. Suzuki, A. Yangisawa, J. Am. Chem. Soc. 1988, 110, $4718-4726$.

[6] L. V. Hijfte, M. Kolb, Tetrahedron 1992, 48, $6393-$ 6402; C. R. Johnson, M. P. Braun, J. Am. Chem. Soc. 1993, 115, $11014-11015$.

[7] L. A. Arnold, R. Naasz, A. J. Minnaard, B. L. Feringa, J. Am. Chem. Soc. 2001, 123, 5841-5842. 
[8] E. Roulland, C. Monneret, J. C. Florent, J. Org. Chem. 2002, 67, 4399-4406.

[9] N. Miyaura, K. Yamada, H. Suginome, A. Suzuki, J. Am. Chem. Soc. 1985, 107, $972-980$; C.F. Lane, Tetrahedron 1976, 32, $981-990$.

[10] K. C. Nicolaou, J. Y. Ramphal, Y. Abe, Synthesis 1989, $898-901$.

[11] Compound 16 was provided by Pharma. Tech. International Inc., New Jersey (USA).

[12] B. H. Lipshutz, Synthesis 1987, 325-341; B. H. Lip- shutz, M. Koerner, D. A. Parker, Tetrahedron Lett. 1987, 28, 945 - 948 .

[13] J. C. Jung, R. Kache, K. K. Vines, Y. S. Zheng, P. Bijoy, M. Valluri, M. A. Avery, J. Org. Chem. 2004, 69, $9269-9284$.

[14] A. Rodriguez, M. Nomen, B. W. Spur, J. J. Godfroid, Eur. J. Org. Chem. 1999, 10, $2655-2662$.

[15] D. D. Perrin, L. F. Armarego, D. R. Perrin, Purification of Laboratory Chemicals, $2^{\text {nd }}$ ed, Pergamon Press, New York, (USA) 1980. 